Artículo científico

Volumen 30(3):821-840. Septiembre-diciembre, 2019

e-ISSN 2215-3608, doi:10.15517/am.v30i3.36625

http://www.revistas.ucr.ac.cr/index.php/agromeso

\title{
Remoción mecánica del material senescente para la recuperación de pasturas $^{1}$
}

\section{Mechanical removal of senescent material for pasture recovery}

\author{
Luis A. Villalobos-Villalobos ${ }^{2}$, Rodolfo WingChing-Jones ${ }^{2}$
}

1 Recepción: 20 de marzo, 2019. Aceptación: 6 de junio, 2019. Este trabajo formó parte del proyecto de investigación 739-B8-178 inscrito en Vicerrectoría de Investigación, denominado "Prácticas de manejo para la recuperación de pasturas". Universidad de Costa Rica, San José, Costa Rica.

2 Universidad de Costa Rica, Escuela de Zootecnia, Centro de Investigación en Nutrición Animal, San José, Costa Rica. luis.villalobosvillalobos@ucr.ac.cr (autor para correspondencia; https://orcid.org/0000-0001-5653-5678),rodolfo.wingching@ucr.ac.cr (https://orcid.org/00000002-8009-2210).

\section{Resumen}

Introducción. La acumulación de material senescente en pasturas tropicales es resultado de la alta producción de biomasa y bajo nivel de aprovechamiento en pastoreo. La remoción de senescencia en pasturas permite que el ganado acceda a estratos bajos durante el pastoreo, lo que genera un rebrote con mayor concentración de nutrientes. Objetivo. El objetivo de este estudio fue evaluar la respuesta de los pastos Estrella africana (Cynodon nlemfuensis Vanderyst.) y Mombasa (Megathyrsus maximus Jacq.) a la remoción de material senescente, con base en sus componentes estructurales, biomasa y contenido de nutrientes. Materiales y métodos. El experimento se llevó a cabo entre abril y agosto de 2017 en dos fincas, una ubicada en Turrialba, Cartago y la otra en San Carlos, Alajuela. Se utilizó un diseño de chi-cuadrado para evaluar la remoción de material senescente versus los respectivos controles. Resultados. La remoción de pasto senescente incrementó la proporción de hoja en ambos pastos. La biomasa producida tuvo un contenido proteico mayor y menos pared celular, siendo a su vez más digestible que el control sin remoción. Los cambios estructurales tuvieron una mayor duración en el pasto Mombasa, lo cual se atribuyó a las diferencias en arquitectura de la planta respecto al pasto estrella. Conclusión. La remoción del pasto senescente es una estrategia para modificar la composición estructural de las pasturas, con lo que se mejora el perfil nutricional de la materia seca producida. El sistema e intensidad de pastoreo pueden contribuir a una menor acumulación de senescencia, y producir un forraje de mayor valor nutricional por un plazo mayor.

Palabras clave: plantas perennes, recuperación de pasturas, senescencia, biomasa, contenido de nutrientes.

\begin{abstract}
Introduction. The accumulation of senescent material in tropical pastures is the result of high biomass production and low level of utilization. By removing senescence pastures, cattle can graze at lower levels, allowing regrowth with higher nutrient content. Objective. The objective of this study was to evaluate the response of African stargrass (Cynodon nlemfuensis Vanderyst.) and Mombasa (Megathyrsus maximus Jacq.) to removal of senescent material based on its structural components, biomass and nutrient content. Materials and methods. This experiment was carried out
\end{abstract}


between April to August 2017 in two farms, one located in Turrialba, Cartago, and the other in San Carlos, Alajuela. A chi-squared design was used to evaluate the removal of senescent material versus the respective controls. Results. Senescence grass removal increased the proportion of leaves in both kinds of grasses. Yielded biomass in senescence material removal plots had higher protein and lower cell wall content, producing more digestible forage. The structural changes had a longer duration in the Mombasa grass, which was attributed to the diffrerences in plant architecture with respect to the Star grass. Conclusion. Removing senescent material from tropical pastures is a strategy to modify the structure and enhance the nutritional value of dry matter yield. Both the type of system and grazing intensity may contribute to reducing senescence accumulation, increasing the nutritive value of forages in the long term.

Keywords: perennial plants, pasture recovery, senescence, biomass, nutrient content.

\section{Introducción}

La senescencia es un proceso biológico adquirido en el proceso evolutivo de las plantas en el cual las hojas son sujetas a cambios fisiológicos y bioquímicos que resultan en una redistribución de nutrientes a otros órganos en desarrollo (Thomas, 2013; Simioni et al., 2014; Woo et al., 2018). La velocidad con la que ocurre dicho proceso está relacionada con factores genéticos (Woo et al., 2018), ambientales y morfológicos (Thomas, 2013). Estados fenológicos como la floración y la producción de semilla sirven como señales para el inicio de senescencia en cereales y otros pastos (Sarath et al., 2014).

La expresión de senescencia en cultivos de importancia agronómica limita la producción de biomasa y modifica su valor nutricional (Wilman y Martins, 1977; Woo et al., 2018). En cultivos anuales como el maíz y el trigo, los nutrientes de las hojas senescentes son movilizados al grano como estructura responsable para lograr perennidad (Sarath et al., 2014), mientras que en cultivos perennes dichos nutrientes se destinan a mayor crecimiento vegetativo (rizomas, estolones, meristemos y raíces) como estrategia principal para evitar estrés y mantenerse viables (Sarath et al., 2014; Sade et al., 2017).

En el caso de pasturas perennes tropicales, la tasa de aparición, elongación y senescencia en hojas, el macollamiento y la composición morfológica, son fundamentales para entender la acumulación de forraje como respuesta de las plantas a un sistema de manejo específico y su relación con el clima (Ramírez-Reynoso et al., 2009; Montagner et al., 2012; Simioni et al., 2014). Entre períodos de defoliación, un porcentaje del forraje se acumulará como senescente (Wilman y Martins, 1977), debido a la relación directa entre la altura de la pastura y la velocidad de senescencia, por lo que especies de pastos con altas tasas de crecimiento tienden a mostrar mayores pérdidas por este proceso (Simioni et al., 2014).

El ciclo de vida de una hoja en un rebrote de pasto está íntimamente relacionado con la hoja sucesiva, así la aparición de una hoja nueva ocurre de forma simultánea con la interrupción de crecimiento de la hoja anterior y la senescencia de la hoja más madura (Simioni et al., 2014). El amarillamiento de las hojas es uno de los signos más evidentes como resultado de la degradación masiva de la clorofila en los cloroplastos (Simioni et al., 2014; Woo et al., 2018). Los pastos tropicales pasan por una etapa de acumulación de hojas hasta un punto de estabilidad, posterior al cual, la aparición de hojas nuevas es compensada por la senescencia de las hojas más maduras y la mayor acumulación de forraje proviene de tallos y material senescente (Ramírez-Reynoso et al., 2009).

El manejo de pasturas tropicales como Mombasa (Megathyrsus maximus Jacq.) puede ser complicado por el tipo de crecimiento, alturas de cosecha y de residuo; características que influyen sobre la calidad (Patiño-Pardo et al., 2018) y persistencia de las pasturas (Wilman y Martins, 1977; Gelley et al., 2017). La reducción en senescencia se puede lograr a través del manejo estratégico basado en la morfo-fisiología del pasto en lugar de intervalos fijos de 
tiempo (Montagner et al., 2012). En países de clima templado se usa el segado como estrategia para reducir la altura del pasto y la biomasa remanente del ciclo de crecimiento anterior (otoño-invierno), previo al inicio del nuevo ciclo de crecimiento (primavera-verano) (Dusseux et al., 2014). Estrategias de este tipo permiten incrementar la uniformidad en pasturas (Tälle et al., 2016) y maximizar la eficiencia de cosecha del forraje producido en sistemas de pastoreo (Simioni et al., 2014).

La escogencia del momento óptimo de cosecha en pasturas se apoya en criterios como la fenología (Donaghy y Fulkerson, 2001), el intervalo y la altura de corte (Wilman y Martins, 1977; Gelley et al., 2017), entre otras. Dichos criterios buscan evitar el deterioro del dosel e incrementar la eficiencia de utilización del forraje producido, sin afectar la persistencia de la pastura (Ramírez-Reynoso et al., 2009). Al disminuir la senescencia se incrementa la disponibilidad de hojas verdes (Montagner et al., 2012), las cuales tienen una concentración de nutrientes mayor que el resto de los órganos de la planta (Paris et al., 2016).

Un mayor porcentaje de hojas en el dosel se ha asociado con mejores índices productivos de los animales en pastoreo, pues se incrementa la digestibilidad y el consumo de materia seca (Paris et al., 2016), permitiendo atenuar los cambios en la disponibilidad y calidad de los forrajes por efecto de las variaciones en las condiciones climáticas (Villalobos y Arce, 2013; Simioni et al., 2014).

Cosechar un forraje con alto valor nutricional, puede además influir sobre el potencial de emisiones de gases de efecto invernadero en sistemas pecuarios. El análisis de producción de gas in vitro (ANKOM, 2012), está estrechamente relacionado con el valor nutricional de los forrajes (Xu et al., 2010; Murillo et al., 2011; Cho et al., 2012; Arce-Ramírez, 2016), por lo que es un indicador adecuado para evaluar el efecto de las estrategias de manejo de pasturas en condiciones tropicales.

La degradación de pasturas tiende a asociarse con factores como la quema, invasión de malezas, plagas y enfermedades, factores climáticos, baja fertilidad de los suelos (Padilla et al., 2009) y el sobrepastoreo. Este último es más una consecuencia de la disminución en productividad como resultado de los demás factores (Villanueva e Ibrahim, 2002; Martínez-Méndez et al., 2016), sin embargo, poco se menciona al subpastoreo como causa de degradación de pasturas en el trópico.

La subutilización de pasturas puede acarrear síntomas de degradación como son la pérdida de vigor de la planta y menor ingreso de luz a estratos bajos (Wilman y Martins, 1977), permitiendo que especies invasoras se establezcan (Martínez-Méndez et al., 2016). En pasturas nativas y semi-nativas se ha visto que el segado genera una homogenización en la vegetación al reducir la competencia inter- e intra-específica (Tälle et al., 2016). Las estrategias de recuperación de pasturas resultan además menos onerosas y alcanzables en plazos menores que el establecimiento de pasturas nuevas (Padilla et al., 2009).

En el trópico, las condiciones climáticas permiten utilizar pastoreo de forma anual (Villalobos et al., 2013), sin embargo, bajo un manejo constante a lo largo del año, la utilización de las pasturas presenta variaciones amplias (Villalobos y Sánchez, 2010; Villalobos et al., 2013). El uso del pastoreo intensivo permite una alta renovación de tejidos, con mayor acumulación de hoja y un control más efectivo sobre la acumulación de tallos (Montagner et al., 2012).

La acumulación de material senescente en pasturas tropicales es un problema de manejo que disminuye su productividad, lo cual puede llevar a la degradación de las mismas. El objetivo de este estudio fue evaluar la respuesta de los pastos estrella africana (Cynodon nlemfuensis Vanderyst.) y Mombasa (Megathyrsus maximus Jacq.) a la remoción del material senescente, con base en sus componentes estructurales, la biomasa y el contenido de nutrientes. 


\section{Materiales y métodos}

\section{Especies de pastos y localidades}

El presente estudio se llevó a cabo entre los meses de abril a agosto de 2017, en dos fincas lecheras, ubicadas en los cantones de Turrialba, Cartago $\left(9^{\circ} 54 \mathrm{~N}, 83^{\circ} 40 \mathrm{O}, 600 \mathrm{msnm}\right)$ y San Carlos, Alajuela $\left(10^{\circ} 22 \mathrm{~N}, 84^{\circ}\right.$ 21, $450 \mathrm{msnm}$ ), y donde se utilizan los pastos estrella africana (Cynodon nlemfuensis Vanderyst.) y Mombasa (Megathyrsus maximus Jacq.) para la alimentación animal, respectivamente. Ambas localidades se clasifican en la zona de vida bosque muy húmedo tropical (bmh-T) según las zonas de vida de Holdridge (1947), las cuales tienen precipitaciones de hasta $4000 \mathrm{~mm}$ anuales y no existe una estación seca bien definida, solo períodos donde las lluvias disminuyen (Quesada, 2007). Ambas fincas utilizan sistemas de pastoreo rotacional con períodos de recuperación de 25 y 16 días, en potreros con áreas promedio de 900 y $3400 \mathrm{~m}^{2}$, para los pastos estrella y Mombasa, respectivamente. La finca de pasto estrella realiza control de malezas manual, no se indica presencia de plagas de importancia y fertilización a un equivalente anual de $150 \mathrm{~kg} \mathrm{~N}^{-1}$ con una presión instantánea de pastoreo promedio de veintidós vacas en ordeño $(450 \mathrm{~kg})$ en 1000 diarios de área de pastoreo $\left(45 \mathrm{~m}^{2}\right.$ animal $\left.^{-1}\right)$. La finca cultivada con pasto Mombasa realiza control de malezas con una mezcla (2,4 D picloram y metsulfurón metil), y fertilización equivalente a $250 \mathrm{~kg} \mathrm{~N} \mathrm{ha}^{-1}$ con una presión instantánea de pastoreo promedio de cuarenta vacas en ordeño $(500 \mathrm{~kg})$ en $5000 \mathrm{~m}^{2}$ diarios de área de pastoreo $\left(125 \mathrm{~m}^{2}\right.$ animal $\left.^{-1}\right)$.

\section{Tratamientos}

Se escogieron dos potreros del pasto estrella en la finca ubicada en Turrialba, con una edad de veinticinco días y se dividieron en cuatro partes $\left(220 \mathrm{~m}^{2}\right.$ cada uno), para un total de ocho parcelas experimentales. De estas, la mitad se cosecharon con una motosegadora autoimpulsada con altura ajustable $(5-10 \mathrm{~cm})$ a una altura de $5 \mathrm{~cm}(\mathrm{E}$ MS), y las otras cuatro se utilizaron como tratamiento control (E-C) de la condición normal sin remover el material senescente acumulado.

Para el pasto Mombasa se escogió un potrero de $3200 \mathrm{~m}^{2}$ en la finca ubicada en San Carlos y se dividió a la mitad de forma longitudinal (dos franjas). Una de las franjas se cosechó con una motoguadaña a una altura de 15 $\mathrm{cm}$, la cual es la altura utilizada por el productor de dicha finca cuando lo hace de forma rutinaria en sus potreros. Cada franja se dividió en cuatro parcelas ( $400 \mathrm{~m}^{2}$ cada una), la franja cosechada fue el tratamiento experimental (M-MG) de remoción de senescencia y la otra mitad el control (M-C). El material cosechado se acordonó y recogió en los potreros de estrella, mientras que para el pasto Mombasa se dejó en campo sin que obstruyera el adecuado rebrote y pastoreo de los animales en los ciclos sucesivos. En el primer muestreo, posterior a la aplicación de los tratamientos, no hubo dificultad en la recolección de las muestras de pasto Mombasa, pues el material dejado se incorporó rápidamente al suelo. Lo anterior se atribuyó a las condiciones particulares de temperatura $\left(>24^{\circ} \mathrm{C}\right)$ y humedad de las zonas donde se ubicaban ambas fincas (Quesada, 2007).

\section{Variables de respuesta evaluadas}

El pasto estrella africana se cosechó el día antes de ingresar los animales a pastoreo (veinticuatro días de recuperación) por medio de un marco de $0,25 \mathrm{~m}^{2}(0,50 \times 0,50 \mathrm{~m})$ a una altura de $5 \mathrm{~cm}$. El pasto Mombasa se cosechó el día previo al ingreso de los animales (quince días de recuperación) con un marco de 0,56 $\mathrm{m}^{2}(0,75 \mathrm{x}$ $0,75 \mathrm{~m}$ ) a una altura de $15 \mathrm{~cm}$.

En ambos pastos se sacó una submuestra de $250 \mathrm{~g}$, la cual se utilizó para composición estructural por medio de la separación en fresco de hojas, tallos y material senescente. Cada componente se secó por aparte a $60{ }^{\circ} \mathrm{C}$ por $48 \mathrm{~h}$ 
con el objetivo de expresar dicha composición en base a la materia seca. El resto de las muestras de cada parcela se secaron a $60{ }^{\circ} \mathrm{C}$ por $48 \mathrm{~h}$ para estimar la producción de biomasa y realizar los análisis nutricionales. Se realizaron cuatro muestreos en cada pasto entre los meses de abril a agosto del 2017.

Las variables evaluadas en los pastos estrella africana y Mombasa fueron la composición estructural (\% hojas, tallos y senescencia), la producción de biomasa $\left(\mathrm{kg} \mathrm{MS} \mathrm{ha}^{-1}\right)$ y el valor nutricional. Los valores de producción de biomasa y composición estructural se combinaron para estimar la producción de cada uno de los componentes estructurales en ambos pastos. El valor nutricional se evaluó por medio de la materia seca (MS) y proteína cruda (PC) (AOAC, 1990), se obtuvo también el contenido de pared celular ( $\alpha$ FDN) (Van-Soest et al., 1991), digestibilidad in vitro de la materia seca (DIVMS) y digestibilidad de la pared celular (DIVFDN) (Van-Soest y Robertson, 1985). Se evaluó además la producción de gas in vitro (PG, $\mathrm{ml} \mathrm{g}^{-1} \mathrm{MS}$ ) con base en la metodología del sistema de producción de gas ANKOM (2012).

\section{Diseño experimental y análisis estadístico}

Se utilizó un arreglo aleatorizado de parcelas divididas con dos tratamientos experimentales (control y remoción de senescencia) en cuatro muestreos con cuatro repeticiones (parcelas) por tratamiento ( $\mathrm{n}=16$ por tratamiento por pasto). Se realizó un análisis de varianza (ANOVA) con la información de biomasa, composición estructural y valor nutricional de ambos pastos, mediante el procedimiento PROC MIXED del paquete estadístico SAS/STAT ${ }^{\circledR}$ 9.3. El modelo incluyó los efectos del tratamiento y las fechas de muestreo como efectos principales y la interacción entre ambos. La fecha de muestreo se consideró como parte del modelo, con el objetivo de evidenciar si los tratamientos de remoción de senescencia tienen un efecto temporal. Se estimó las diferencias significativas $(\mathrm{p}<0,05)$ entre medias de los tratamientos y las fechas de muestreo para todas las variables en base al error tipo III, mediante el procedimiento PROC GLIMMIX del programa estadístico SAS® 9.3.

\section{Resultados}

\section{Composición estructural y relación hoja:tallo (RHT)}

El pasto estrella africana mostró una proporción de material senescente significativamente menor $(\mathrm{p}<0,05)$ con la remoción de senescencia respecto al control (9,65 vs 19,7\%), lo cual hizo que la proporción de hoja incrementara $(42,6$ vs $34,3 \%$, Figura 1$)$ de forma significativa $(\mathrm{p}<0,05)$. El tratamiento, la fecha de muestreo y la interacción de ambos influyeron sobre las proporciones encontradas en ambos componentes del pasto estrella (Cuadro 1).

El pasto Mombasa tuvo una proporción de hoja significativamente mayor $(\mathrm{p}<0,05)$ que el control, casi del doble (63,5 vs 36,1\%), por efecto del tratamiento. El material senescente en Mombasa disminuyó significativamente $(\mathrm{p} \leq 0,0001)$, siendo una cuarta parte de lo que representaba en las parcelas sin remoción ( 8,0 vs $32,5 \%)$. Los efectos principales de tratamiento y fecha de muestreo, tuvieron efectos significativos sobre ambos componentes en el pasto Mombasa (Cuadro 1).

La remoción de material senescente no afectó la proporción de tallo, y mostró en ambos pastos valores similares entre el tratamiento aplicado y las parcelas control (Figura 1). En el pasto estrella africana hubo un efecto significativo de la fecha de muestreo (Cuadro 1). La relación hoja:tallo fue mayor en los pastos estrella africana $(\mathrm{p}<0,05)$ y Mombasa $(\mathrm{p} \leq 0,0001)$, con remoción del material senescente respecto a sus respectivos controles (Figura 2). Dicho indicador se vio afectado por el tratamiento y la fecha de muestreo en ambos pastos (Cuadro 1). 


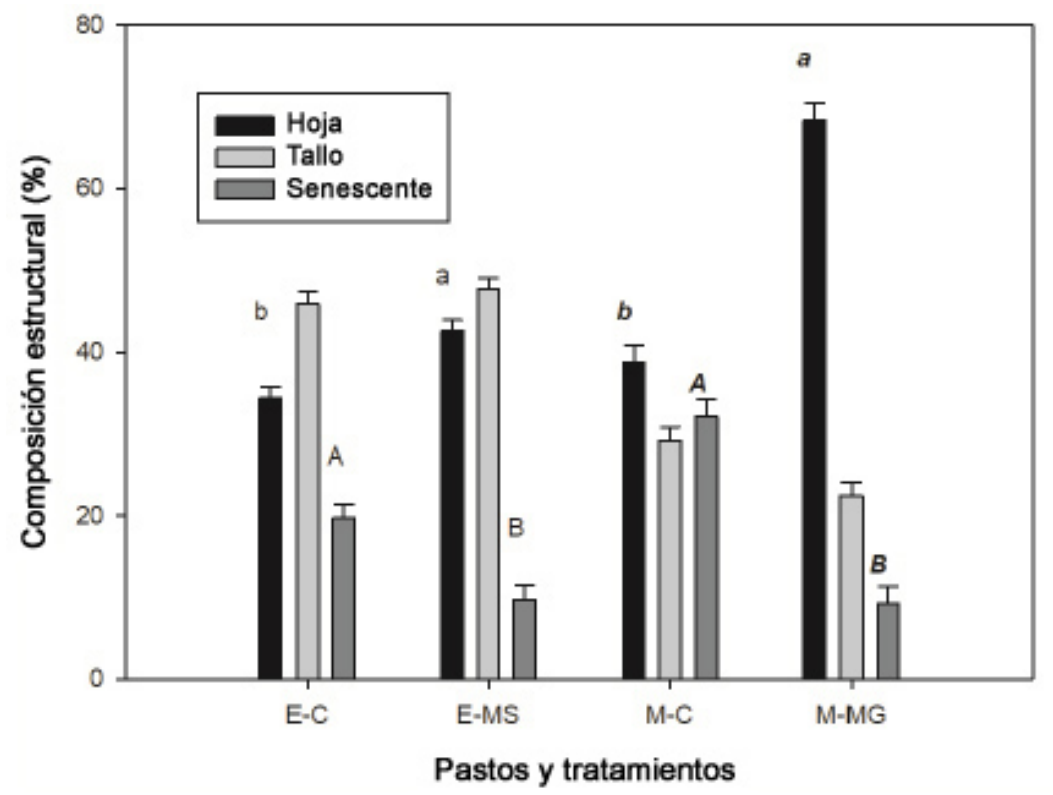

Figura 1. Componentes estructurales de los pastos estrella africana (Cynodon nlemfuensis Vanderyst.) y Mombasa (Megathyrsus maximus Jacq.), en Turrialba y San Carlos, respectivamente. Costa Rica. 2017.

$\mathrm{E}-\mathrm{C}=$ estrella: control, E-MS= estrella: tratamiento experimental, $\mathrm{M}-\mathrm{C}=$ Mombasa: control, $\mathrm{M}-\mathrm{MG}=\mathrm{Mombasa}$ : tratamiento experimental. Letras diferentes entre medias de los componentes senescente (mayúsculas) y hoja (minúsculas) fueron differentes en base al error tipo III

Figure 1. Structural components of African stargrass (Cynodon nlemfuensis Vanderyst.) and Mombasa (Megathyrsus maximus Jacq.) in Turrialba and San Carlos, respectively. Costa Rica. 2017.

$\mathrm{E}-\mathrm{C}=$ stargrass: control. $\mathrm{E}-\mathrm{MS}=$ stargrass: experimental treatment. $\mathrm{M}-\mathrm{C}=\mathrm{Mombas}$ : control, $\mathrm{M}-\mathrm{MG}=\mathrm{Mombasa}$ : experimental treatment. Different letters between means of senescent material (uppercase) and leaf (lowercase) were different based on error type III.

Cuadro 1. Análisis de varianza de los efectos principales e interacciones incluidas en el modelo estadístico para los componentes estructurales, relación hoja: tallo y biomasa de los pastos Estrella africana (Cynodon nlemfuensis Vanderyst.) y Mombasa (Megathyrsus maximus Jacq.) en Turrialba y San Carlos, respectivamente. Costa Rica, 2017.

Table 1. Analysis of variance of the main effects and interactions included in the statistical model for structural components, leaf-tostem ratio and biomass of African stargrass (Cynodon nlemfuensis Vanderyst.) and Mombasa (Megathyrsus maximus Jacq.) in Turrialba and San Carlos, respectively. Costa Rica, 2017.

\begin{tabular}{|c|c|c|c|c|}
\hline Variable & Especie & Tratamiento $(\mathrm{T})^{1}$ & Muestreo (M) & TxM \\
\hline \multirow{2}{*}{$\%$ hoja } & Estrella & $*$ & $* *$ & $*$ \\
\hline & Mombasa & $* *$ & $*$ & n.s. \\
\hline \multirow{2}{*}{$\%$ tallo } & Estrella & n.s. & $* *$ & n.s. \\
\hline & Mombasa & n.s. & n.s. & n.s. \\
\hline \multirow{2}{*}{$\%$ senescente } & Estrella & $*$ & $* *$ & $* *$ \\
\hline & Mombasa & $* *$ & $*$ & n.s. \\
\hline \multirow{2}{*}{ Relación hoja:tallo } & Estrella & $*$ & $* *$ & n.s. \\
\hline & Mombasa & $* *$ & $*$ & n.s. \\
\hline \multirow{2}{*}{ Biomasa } & Estrella & n.s. & $*$ & n.s. \\
\hline & Mombasa & $* *$ & $*$ & $*$ \\
\hline
\end{tabular}

${ }^{1}$ n.s: no significativo / ${ }^{1}$ n.s: non-significant. $*$ significativo $(\mathrm{p}<0,05) / *$ significant $(\mathrm{p}<0.05)$. ** altamente significativo $(\mathrm{p} \leq 0,0001) /$ ** highly significant $(\mathrm{p} \leq 0.0001)$. 


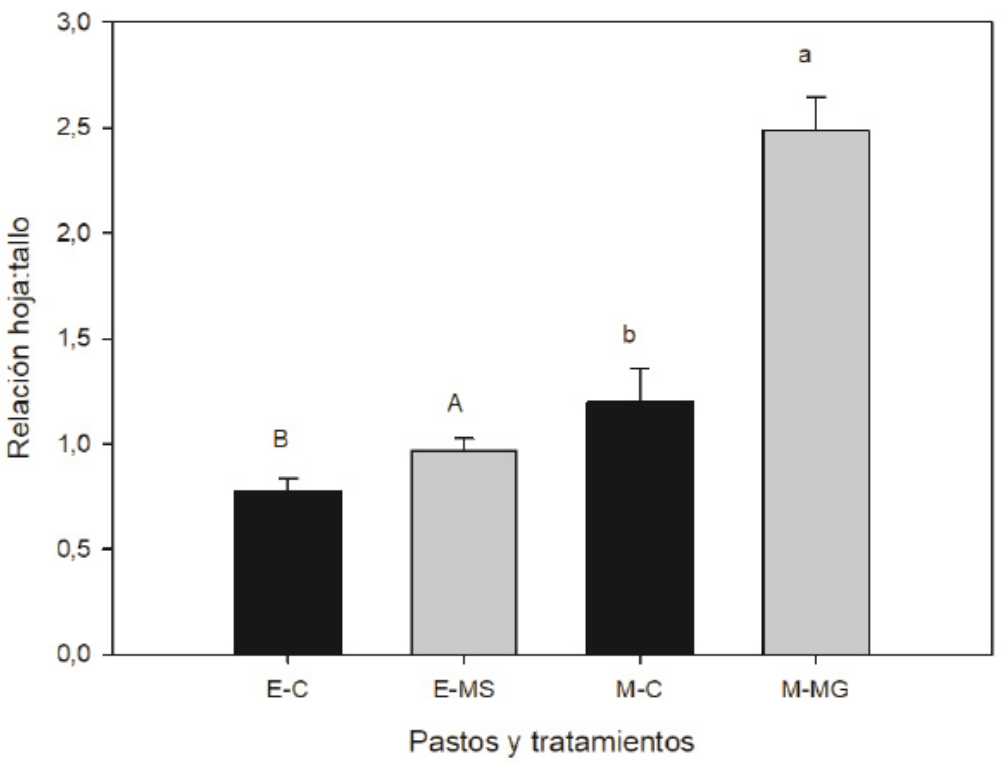

Figura 2. Relación hoja:tallo de pastos estrella africana (Cynodon nlemfuensis Vanderyst.) y Mombasa (Megathyrsus maximus Jacq.) en Turrialba y San Carlos, respectivamente. Costa Rica. 2017.

$\mathrm{E}-\mathrm{C}=$ estrella: control, E-MS= estrella: tratamiento experimental, $\mathrm{M}-\mathrm{C}=$ Mombasa: control, M-MG=Mombasa: tratamiento experimental. Letras diferentes entre medias de los componentes senescente (mayúsculas) y hoja (minúsculas) fueron differentes en base al error tipo III

Figure 2. Leaf-to-stem ratio of African stargrass (Cynodon nlemfuensis Vanderyst.) and Mombasa (Megathyrsus maximus Jacq.) in Turrialba and San Carlos, respectively. Costa Rica. 2017.

$\mathrm{E}-\mathrm{C}=$ stargrass: control. $\mathrm{E}-\mathrm{MS}=$ stargrass: experimental treatment. $\mathrm{M}-\mathrm{C}=\mathrm{Mombasa}$ : control, $\mathrm{M}-\mathrm{MG}=\mathrm{Mombasa}$ : experimental treatment. Different letters between means of senescent material (uppercase) and leaf (lowercase) were different based on error type III.

\section{Producción de biomasa}

La producción de biomasa se vio afectada por el tratamiento, el muestreo y la interacción de ambos en el pasto Mombasa, mientras que solo la época de muestreo tuvo un efecto significativo sobre la producción de biomasa del pasto estrella (Cuadro 1). El pasto Mombasa tuvo en promedio en las parcelas control una producción de biomasa equivalente a tres veces la cantidad de materia seca disponible en el tratamiento de remoción; mientras que el pasto estrella africana tuvo valores similares bajo las dos condiciones de manejo (Figura 3). Este último mostró diferencias significativas entre muestreos, el primer muestreo (2906 kg MS ha-1) fue diferente a los demás (5229 $\mathrm{kg} \mathrm{MS} \mathrm{ha-1}$ promedio). La producción de biomasa en el pasto Mombasa no mostró una disminución marcada del primer muestreo (8008 $\mathrm{kg} \mathrm{MS} \mathrm{ha}^{-1}$ ) a los restantes (8958 $\mathrm{kg} \mathrm{MS} \mathrm{ha}^{-1}$ promedio).

Al combinar los datos de biomasa con los componentes estructurales, se pudo observar que la remoción de la pastura senescente favoreció una menor acumulación de material senescente, con una disminución de dicho componente en $68 \%$ y $91 \%$, en pastos estrella y Mombasa, respectivamente (Figura 4). En el pasto Mombasa la remoción de la pastura senescente disminuyó la producción de hojas y tallos en $35 \%$ y $67 \%$, respectivamente.

La producción de biomasa del pasto Mombasa al remover la pastura senescente se consideró aún alta, representando más de cien toneladas de materia seca por hectárea anualmente (Cuadro 2), mientras el control tuvo una producción cercana a tres veces la de este último. En el pasto estrella africana, el tratamiento produjo sesenta toneladas de materia seca anualmente, siendo el control levemente superior en su producción de biomasa anual. 


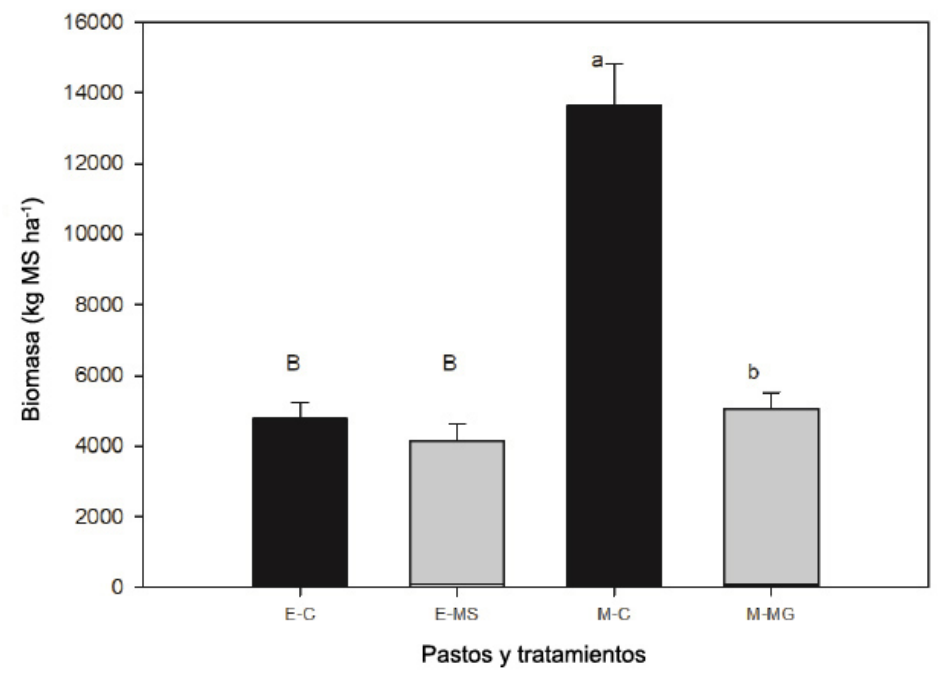

Figura 3. Producción de biomasa (kg MS ha ${ }^{-1}$ ) de pastos estrella africana (Cynodon nlemfuensis Vanderyst.) y Mombasa (Megathyrsus maximus Jacq.) en Turrialba y San Carlos, respectivamente, Costa Rica. 2017.

$\mathrm{E}-\mathrm{C}=$ estrella: control, $\mathrm{E}-\mathrm{MS}=$ estrella: tratamiento experimental, $\mathrm{M}-\mathrm{C}=$ Mombasa: control, $\mathrm{M}-\mathrm{MG}=\mathrm{Mombasa}$ : tratamiento experimental. Letras diferentes entre medias de los componentes senescente (mayúsculas) y hoja (minúsculas) fueron differentes en base al erro tipo III

Figure 3. Biomass production (kg MS ha ${ }^{-1}$ ) of African stargrass (Cynodon nlemfuensis Vanderyst.) and Mombasa (Megathyrsus maximus Jacq.) in Turrialba and San Carlos, respectively, Costa Rica. 2017.

$\mathrm{E}-\mathrm{C}=$ stargrass: control. E-MS= stargrass: experimental treatment. $\mathrm{M}-\mathrm{C}=\mathrm{Mombasa}$ : control, $\mathrm{M}-\mathrm{MG}=\mathrm{Mombasa}$ : experimental treatment. Different letters between means of senescent material (uppercase) and leaf (lowercase) were different based on error type III.

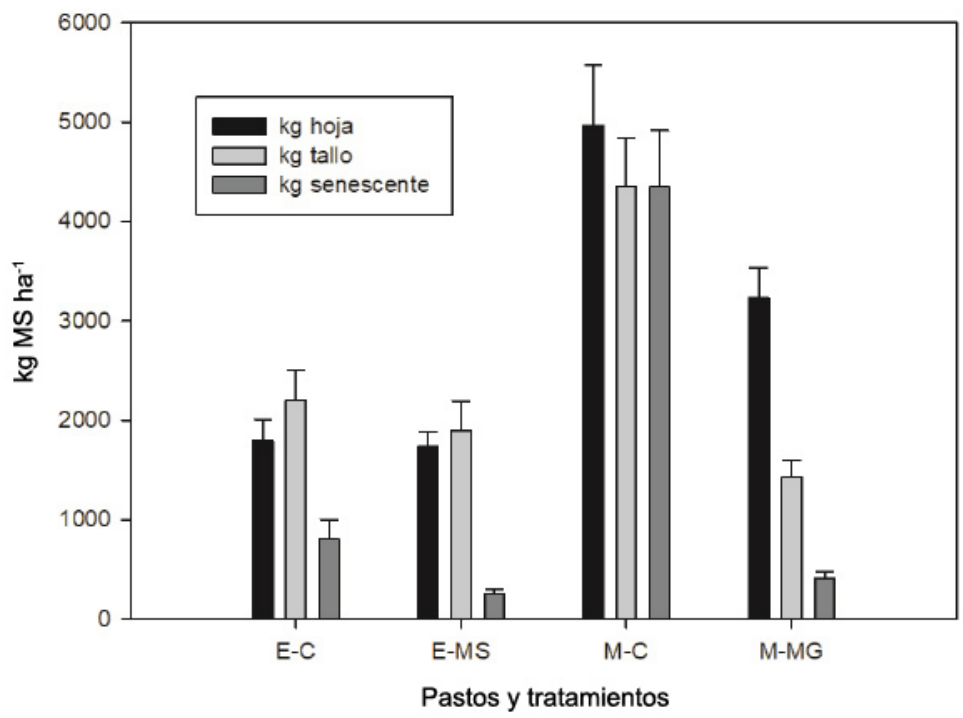

Figura 4. Producción de hojas, tallos y material senescente $\left(\mathrm{kg} \mathrm{MS} \mathrm{ha}^{-1}\right)$ de pastos de los pastos estrella africana (Cynodon nlemfuensis Vanderyst.) y Mombasa (Megathyrsus maximus Jacq.) en Turrialba y San Carlos, respectivamente. Costa Rica. 2017.

E-C= estrella: control, E-MS = estrella: tratamiento experimental, $\mathrm{M}-\mathrm{C}=$ Mombasa: control, $\mathrm{M}-\mathrm{MG}=$ Mombasa: tratamiento experimental

Figure 4. Production of leaves, stems and senescent material yields (kg MS ha ${ }^{-1}$ ) of African stargrass (Cynodon nlemfuensis Vanderyst.) and Mombasa (Megathyrsus maximus Jacq.) in Turrialba and San Carlos, respectively, Costa Rica. 2017.

$\mathrm{E}-\mathrm{C}=$ stargrass: control. $\mathrm{E}-\mathrm{MS}=$ stargrass: experimental treatment. $\mathrm{M}-\mathrm{C}=\mathrm{Mombas}$ : control, $\mathrm{M}-\mathrm{MG}=\mathrm{Mombasa}$ : experimental treatment. 
Cuadro 2. Producción de biomasa promedio por ciclo y anual para los pastos estrella africana (Cynodon nlemfuensis Vanderyst.) y Mombasa (Megathyrsus maximus Jacq.) en Turrialba y San Carlos, respectivamente. Costa Rica. 2017.

Table 2. Average biomass production per cycle and annual for African stargrass (Cynodon nlemfuensis Vanderyst.) and Mombasa (Megathyrsus maximus Jacq.) in Turrialba and San Carlos, respectively. Costa Rica. 2017.

\begin{tabular}{|c|c|c|c|}
\hline Especie & Tratamiento* & $\begin{array}{c}\text { Producción de biomasa } \\
\left(\mathrm{kg} \mathrm{MS} \mathrm{ha}^{-1} \text { ciclo }^{-1}\right)\end{array}$ & $\begin{array}{l}\text { Producción de biomasa } \\
\text { anual (t MS ha-1 año-1) }\end{array}$ \\
\hline \multirow{2}{*}{ Estrella africana } & E-C & 4796 & 70 \\
\hline & E-MS & 4154 & 60 \\
\hline \multirow{2}{*}{ Mombasa } & $\mathrm{M}-\mathrm{C}$ & 13.661 & 290 \\
\hline & M-MG & 5063 & 108 \\
\hline
\end{tabular}

$\mathrm{E}-\mathrm{C}=$ estrella: control. $\mathrm{E}-\mathrm{MS}=$ estrella: tratamiento experimental. $\mathrm{M}-\mathrm{C}=\mathrm{Mombasa}$ : control. $\mathrm{M}-\mathrm{MG}=\mathrm{Mombasa}$ : tratamiento experimental $/ \mathrm{E}-\mathrm{C}=$ stargrass: control. $\mathrm{E}-\mathrm{MS}=$ stargrass: experimental treatment. $\mathrm{M}-\mathrm{C}=$ Mombasa: control, $\mathrm{M}-\mathrm{MG}=\mathrm{Mombasa}$ : experimental treatment.

\section{Valor nutricional}

\section{Materia seca $(M S)$}

El contenido de materia seca mostró efectos significativos del muestreo en ambos pastos (Cuadro 3), mientras que solo en el pasto Mombasa el tratamiento de remoción de la pastura senescente mostró contenidos significativamente menores $(\mathrm{p}<0,05)$ con respecto al control (Figura 5).

Cuadro 3. Análisis de varianza de los efectos principales e interacciones incluidas en el modelo estadístico para las variables de valor nutricional, en los pastos estrella africana (Cynodon nlemfuensis Vanderyst.) y Mombasa (Megathyrsus maximus Jacq.) en Turrialba y San Carlos, respectivamente. Costa Rica. 2017.

Table 3. Analysis of variance of the main effects and interactions included in the statistical model for the nutritional traits of African stargrass (Cynodon nlemfuensis Vanderyst.) and Mombasa (Megathyrsus maximus Jacq.) in Turrialba and San Carlos, respectively. Costa Rica. 2017.

\begin{tabular}{|c|c|c|c|c|}
\hline Variable* & Especie & ${\text { Tratamiento }(\mathrm{T})^{1}}^{1}$ & Muestreo (M) & TxM \\
\hline \multirow{2}{*}{ MS } & Estrella africana & n.s. & $*$ & n.s. \\
\hline & Mombasa & $*$ & $*$ & n.s. \\
\hline \multirow{2}{*}{$\mathrm{PC}$} & Estrella africana & $*$ & $*$ & $* *$ \\
\hline & Mombasa & $* *$ & $*$ & n.s. \\
\hline \multirow{2}{*}{$\alpha \mathrm{FDN}$} & Estrella africana & $* *$ & $*$ & $*$ \\
\hline & Mombasa & $* *$ & n.s. & $*$ \\
\hline \multirow{2}{*}{ DIVFDN } & Estrella africana & $*$ & $* *$ & $*$ \\
\hline & Mombasa & $* *$ & $*$ & $*$ \\
\hline \multirow{2}{*}{ DIVMS } & Estrella africana & $*$ & n.s. & n.s. \\
\hline & Mombasa & $* *$ & $*$ & $*$ \\
\hline \multirow{2}{*}{ PG } & Estrella africana & n.s. & $* *$ & n.s. \\
\hline & Mombasa & $* *$ & $*$ & n.s. \\
\hline
\end{tabular}

${ }^{1}$ n.s: no significativo / non-significant. *: significativo / significant $(\mathrm{p}<0,05)$. **: altamente significativo / highly significant ( $\left.\mathrm{p} \leq 0.0001\right)$. MS: materia seca / dry matter. PC: proteína cruda / crude protein. $\alpha$ FDN: fibra detergente neutra / neutral detergent fiber. DIVFDN: digestibilidad in vitro de la fibra / in vitro fiber digestibility. DIVMS: digestibilidad in vitro de la materia seca / in vitro dry matter digestibility. PG: producción de gas / gas production. 


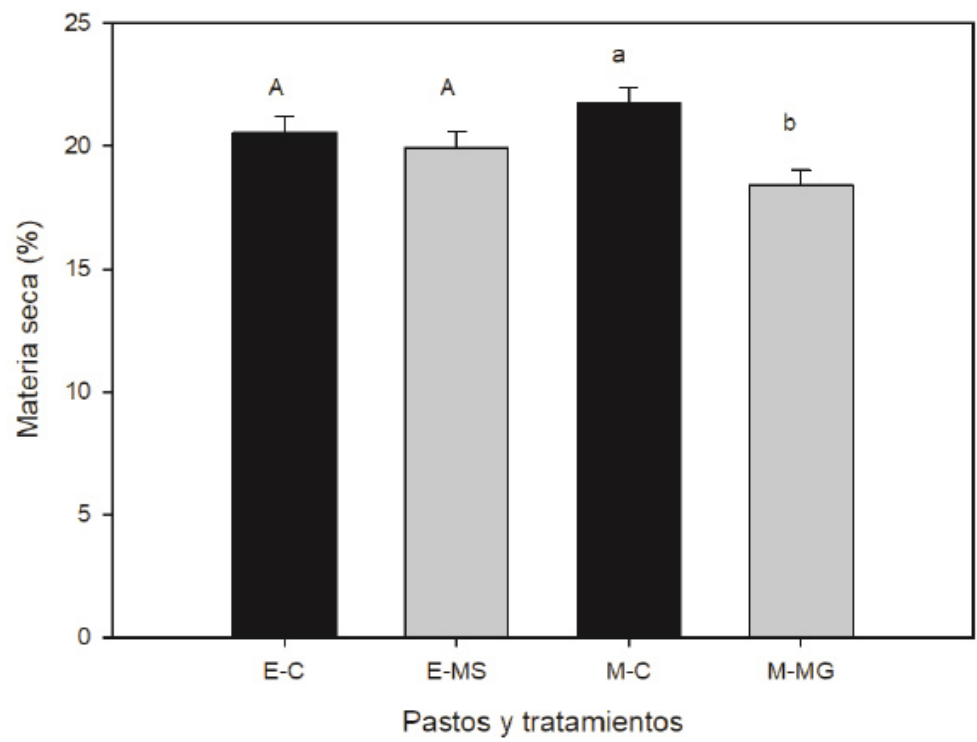

Figura 5. Contenido de materia seca de los pastos estrella africana (Cynodon nlemfuensis Vanderyst.) y Mombasa (Megathyrsus maximus Jacq.) en Turrialba y San Carlos, respectivamente. Costa Rica. 2017.

$\mathrm{E}-\mathrm{C}=$ estrella: control, E-MS= estrella: tratamiento experimental, $\mathrm{M}-\mathrm{C}=$ Mombasa: control, $\mathrm{M}-\mathrm{MG}=\mathrm{Mombasa}$ : tratamiento experimental. Letras diferentes entre medias de los tratamientos en estrella (mayúsculas) y Mombasa (minúsculas) fueron diferentes en base al error tipo III.

Figure 5. Dry matter content of African stargrass (Cynodon nlemfuensis Vanderyst.) and Mombasa (Megathyrsus maximus Jacq.) in Turrialba and San Carlos, respectively. Costa Rica. 2017.

$\mathrm{E}-\mathrm{C}=$ stargrass: control. $\mathrm{E}-\mathrm{MS}=$ stargrass: experimental treatment. $\mathrm{M}-\mathrm{C}=\mathrm{Mombasa}$ : control, $\mathrm{M}-\mathrm{MG}=\mathrm{Mombasa}$ : experimentall treatment. Different letters between means in stargrass (uppercase) and Mombasa (lowercase) were different based on error type III.

\section{Proteína cruda $(P C)$}

El contenido de proteína cruda se vio afectado por el tratamiento y el muestreo en ambos pastos y por la interacción de ambos en el pasto estrella africana (Cuadro 3). La remoción de la pastura senescente favoreció un contenido proteico mayor en ambos pastos (Figura 6).

\section{Contenido de pared celular ( $\alpha F D N)$}

Al contenido de pared celular lo afectó el tratamiento de remoción de la pastura senescente y su interacción con la fecha de muestreo en ambos pastos, y por el muestreo en estrella africana (Cuadro 3). El contenido de pared celular en este último fue significativamente menor con la remoción de la pastura senescente $(62,52 \%)$ con respecto al control $(67,21 \%$ ) (Figura 7$)$. El pasto Mombasa mostró el mismo comportamiento en pared celular, siendo significativamente menor con la remoción de la pastura senescente $(66,52 \%)$ versus el control $(72,12 \%)$.

Digestibilidad in vitro de la pared celular (DIVFDN) y de la materia seca (DIVMS)

En el modelo utilizado el tratamiento, la fecha de muestreo y la interacción entre ambos influyeron sobre la digestibilidad de la pared celular en ambos pastos (Cuadro 3). La pared celular fue más digestible en las parcelas 


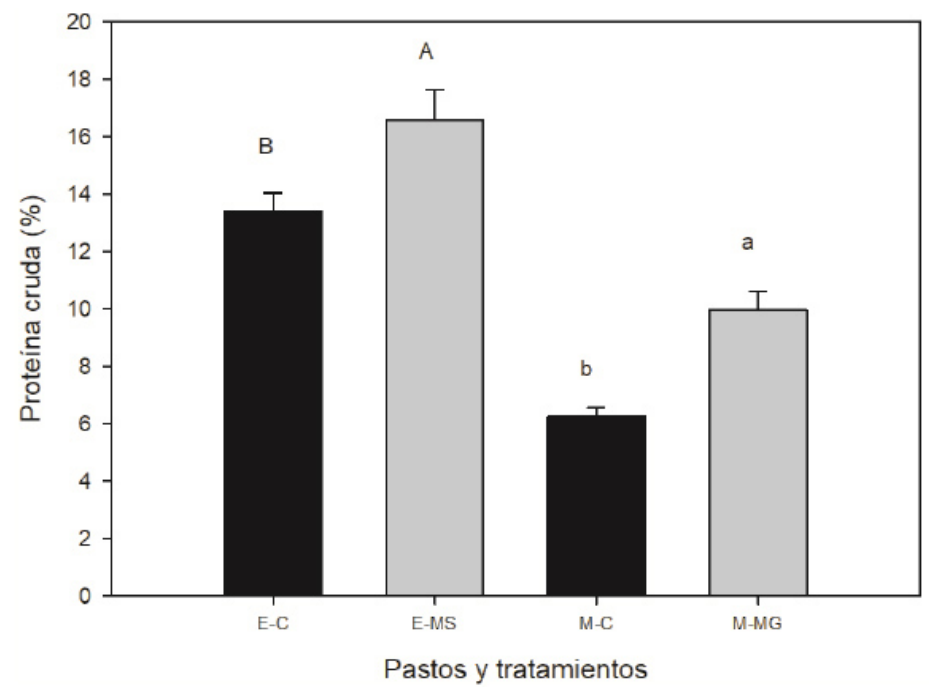

Figura 6. Contenido de proteína cruda de los pastos estrella africana (Cynodon nlemfuensis Vanderyst.) y Mombasa (Megathyrsus maximus Jacq.) en Turrialba y San Carlos, respectivamente. Costa Rica. 2017.

$\mathrm{E}-\mathrm{C}=$ estrella: control, E-MS= estrella: tratamiento experimental, $\mathrm{M}-\mathrm{C}=$ Mombasa: control, $\mathrm{M}-\mathrm{MG}=\mathrm{Mombasa}$ : tratamiento experimental. Letras diferentes entre medias de los componentes estrella (mayúsculas) y Mombasa (minúsculas) fueron differentes en base al error tipo III.

Figure 6. Crude protein content of African stargrass (Cynodon nlemfuensis Vanderyst.) and Mombasa (Megathyrsus maximus Jacq.) in Turrialba and San Carlos, respectively. Costa Rica. 2017.

$\mathrm{E}-\mathrm{C}=$ stargrass: control. $\mathrm{E}-\mathrm{MS}=$ stargrass: experimental treatment. $\mathrm{M}-\mathrm{C}=$ Mombasa: control, $\mathrm{M}-\mathrm{MG}=\mathrm{Mombasa}$ : experimental treatment. Different letters between means in stargrass (uppercase) andMombasa (lowercase) were different based on error type III.

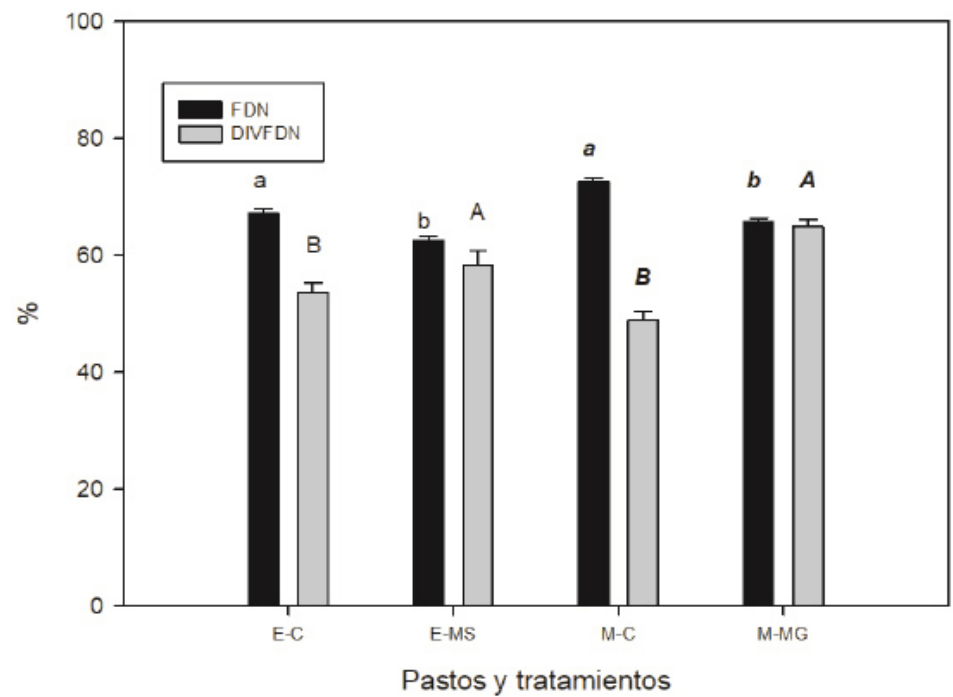

Figura 7. Contenido de pared celular y su digestibilidad de los pastos estrella africana (Cynodon nlemfuensis Vanderyst.) y Mombasa (Megathyrsus maximus Jacq.) en Turrialba y San Carlos, respectivamente. Costa Rica. 2017.

$\mathrm{E}-\mathrm{C}=$ estrella: control, E-MS= estrella: tratamiento experimental, $\mathrm{M}-\mathrm{C}=$ Mombasa: control, M-MG= Mombasa: tratamiento experimental. Letras diferentes entre medias de la fibra (mayúsculas) y digestivilidad de la fibra(minúsculas) fueron differentes en base al error tipo III.

Figure 7. Cell wall content and its digestibility of African stargrass (Cynodon nlemfuensis Vanderyst.) and Mombasa (Megathyrsus maximus Jacq.) in Turrialba and San Carlos, respectively. Costa Rica. 2017.

$\mathrm{E}-\mathrm{C}=$ stargrass: control. $\mathrm{E}-\mathrm{MS}=$ stargrass: experimental treatment. $\mathrm{M}-\mathrm{C}=\mathrm{Mombasa}$ : control, $\mathrm{M}-\mathrm{MG}=\mathrm{Mombasa}$ : experimental treatment. Different letters between means of fiber (uppercase) and fiber digestibility (lowercase) were different based on error type III. 
donde se aplicó el tratamiento de remoción de la pastura senescente (Figura 7), siendo dicha diferencia mayor en el pasto Mombasa $(12,9 \%)$ con respecto al pasto estrella africana $(4,70 \%)$.

La digestibilidad de la materia seca estuvo afectada por el tratamiento de remoción de la pastura senescente en ambos pastos y por la fecha de muestreo y la interacción de ambos en el pasto Mombasa (Cuadro 3). Al igual que ocurrió con las demás variables nutricionales evaluadas, el tratamiento de remoción de la pastura senescente mejoró la digestibilidad de la materia seca en ambos pastos (Figura 8).

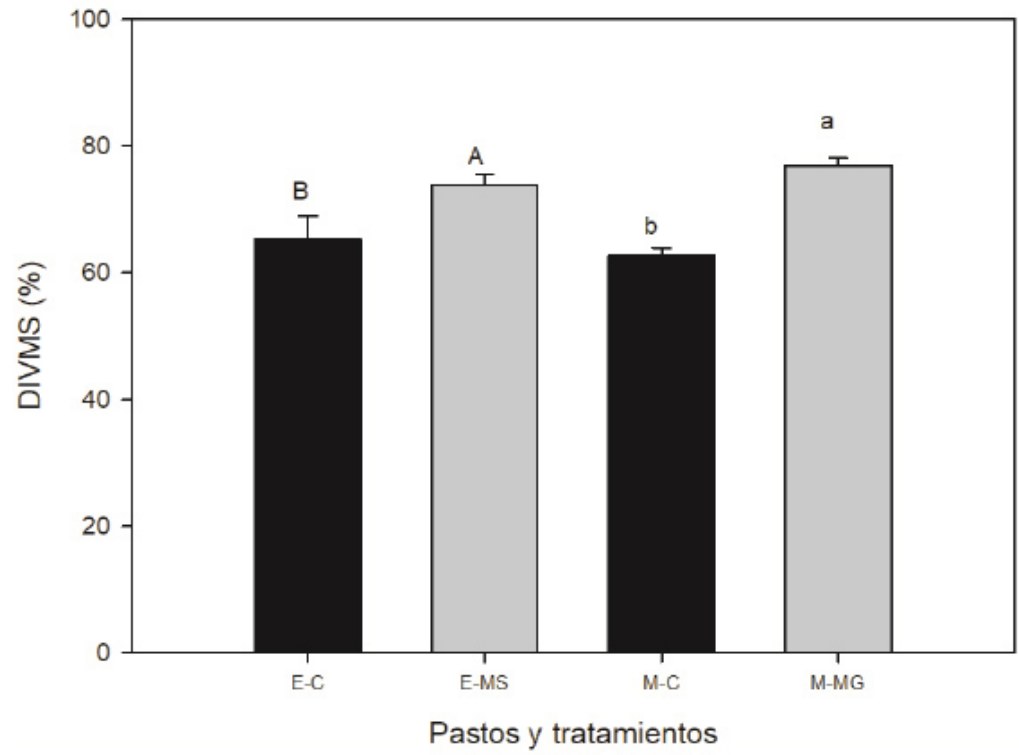

Figura 8. Digestibilidad in vitro de la materia seca de los pastos estrella africana (Cynodon nlemfuensis Vanderyst.) y Mombasa (Megathyrsus maximus Jacq.) en Turrialba y San Carlos, respectivamente. Costa Rica. 2017.

$\mathrm{E}-\mathrm{C}=$ estrella: control, E-MS= estrella: tratamiento experimental, $\mathrm{M}-\mathrm{C}=$ Mombasa: control, $\mathrm{M}-\mathrm{MG}=\mathrm{Mombasa}$ : tratamiento experimental . Letras diferentes entre medias de los tratamientos estrella (mayúsculas) y Mombasa(minúsculas) fueron differentes en base al error tipo III.

Figure 8. In-vitro dry matter digestibility of African stargrass (Cynodon nlemfuensis Vanderyst.) and Mombasa (Megathyrsus maximus Jacq.) in Turrialba and San Carlos, respectively. Costa Rica. 2017.

$\mathrm{E}-\mathrm{C}=$ stargrass: control. $\mathrm{E}-\mathrm{MS}=$ stargrass: experimental treatment. $\mathrm{M}-\mathrm{C}=$ Mombasa: control, $\mathrm{M}-\mathrm{MG}=\mathrm{Mombasa}$ : experimental treatment. Different letters between means in stargrass (uppercase) and Mombasa (lowercase) were different based on error type III.

\section{Producción de gas in vitro de la materia seca $(P G)$}

La producción de gas in vitro tuvo, en el modelo utilizado, un efecto significativo de la fecha de muestreo en ambos pastos y del tratamiento en el pasto Mombasa (Cuadro 3). El pasto estrella africana tuvo valores similares de producción de gas en ambos tratamientos (Figura 9), mientras que el pasto Mombasa tuvo valores mayores en el tratamiento de remoción de senescencia (77,41 vs $54,20 \mathrm{ml} \mathrm{g}^{-1} \mathrm{MS}$ ). 




Figura 9. Producción de gas in vitro de la materia seca de los pastos Estrella africana (Cynodon nlemfuensis Vanderyst.) y Mombasa (Megathyrsus maximus Jacq.) en Turrialba y San Carlos, respectivamente. Costa Rica. 2017.

$\mathrm{E}-\mathrm{C}=$ estrella: control, $\mathrm{E}-\mathrm{MS}=$ estrella: tratamiento experimental, $\mathrm{M}-\mathrm{C}=$ Mombasa: control, $\mathrm{M}-\mathrm{MG}=\mathrm{Mombasa}$ : tratamiento experimental. Letras diferentes entre medias de los tratamientos estrella (mayúsculas) y Mombasa (minúsculas) fueron differentes en base al error tipo III.

Figure 9. In vitro gas production of dry matter from African stargrass (Cynodon nlemfuensis Vanderyst.) and Mombasa (Megathyrsus maximus Jacq.) in Turrialba and San Carlos, respectively. Costa Rica. 2017.

$\mathrm{E}-\mathrm{C}=$ stargrass: control. $\mathrm{E}-\mathrm{MS}=$ stargrass: experimental treatment. $\mathrm{M}-\mathrm{C}=$ Mombasa: control, $\mathrm{M}-\mathrm{MG}=\mathrm{Mombasa}$ : experimental treatment. Different letters between means in stargrass (uppercase) and Mombasa (lowercase) were different based on error type III.

\section{Discusión}

El efecto de la remoción de material senescente fue de diferente magnitud en ambos pastos, las proporciones de hoja y material senescente se vieron modificadas. En pastos como Brachiaria ruziziensis se ha encontrado una proporción de hojas de $73 \%$ y $47 \%$ con edades de cosecha de dos y cinco semanas, respectivamente (Simioni et al., 2014). En cambio, en pasto Mombasa se determinó una disminución en la proporción de hojas (75 a 56 \%) y un aumento en el material senescente $(1$ a $8 \%$ ) al aumentar la edad de cosecha de tres a siete semanas (RamírezReynoso et al., 2009).

En pasto Tanzania se encontró una menor proporción de hoja $(60,3-62,2 \%)$ y mayor de tallo $(17,9-21,4 \%)$ y senescencia $(18,4 \%)$ en un sistema de rotación con días fijos (30-35 días) con respecto a las proporciones de hoja (71,6-79,0 \%), tallo (12,0-16,6\%) y pasto senescente (11,7 \%), bajo un ajuste variable en base a la altura de ingreso $(70 \mathrm{~cm})$ a la pastura, cuyos períodos de recuperación siempre fueron menores (23-31 d) (Euclides et al., 2014). En el presente estudio, el pasto Mombasa mantuvo una proporción de hoja mayor a lo largo de los cuatro muestreos, mientras que el pasto Estrella tuvo una proporción de hoja similar a la inicial en el cuarto muestreo. 
Los criterios utilizados en el manejo de pasturas deben lograr que el momento de cosecha se realice en un punto en el que la biomasa y la calidad del forraje producido llenen las metas del sistema productivo (Gelley et al., 2017), y mantengan una composición botánica deseada (Simioni et al., 2014). Asimismo, una menor presencia de material senescente incrementa la penetración de luz en la cepa, lo cual promueve el rebrote en el ciclo siguiente (Montagner et al., 2012).

En este estudio, los días de rotación no se modificaron, lo cual pudo afectar la respuesta productiva de ambos pastos, debido a los cambios climatológicos en ambas zonas (Quesada, 2007). En el pasto estrella africana se encontró una proporción de tallo menor en el primer muestreo (34,1\%) con respecto a los restantes tres $(51,1 \%$ promedio), lo cual se atribuyó a un efecto temporal del tratamiento y de la época del año, tal como se ha visto en otros estudios (Montagner et al., 2012; Gelley et al., 2017). Existen factores climáticos que pueden favorecer una mayor producción de hoja en un ciclo de rotación (Gelley et al., 2017), lo cual pudo influir en que los componentes estructurales se igualaran en los siguientes muestreos.

La relación hoja:tallo fue menor a 1 en el pasto estrella africana en el control y en el tratamiento de remoción de la pastura senescente $(0,77$ vs 0,96$)$. Esto puede atribuirse a que el pasto estrella, bajo el manejo utilizado en la finca, tuvo una velocidad de senescencia similar a la tasa de aparición de hojas nuevas, mostrando un número de hojas constante por rebrote (Simioni et al., 2014). La relación hoja:tallo en pasto estrella africana en este estudio fue mayor a la reportada por Paris et al. (2016), quienes manejaron diferentes niveles de producción de hojas, con valores entre 0,38 a 0,68 .

En el pasto Mombasa la relación hoja:tallo incrementó a más del doble con la remoción de la pastura senescente respecto al control (2,49 vs 1,20). Se ha encontrado relaciones hoja:tallo de 3,1 a 1,6 en época lluviosa al aumentar la edad de cosecha de tres a siete semanas (Ramírez-Reynoso et al., 2009), mientras en pasto Tanzania se han encontrado relaciones hoja:tallo superiores $(4,3-6,6)$ bajo un sistema de rotación variable basado en la altura, con respecto a los de un sistema de rotación con días fijos (2,8-3,5) (Euclides et al., 2014).

El aumento en la proporción de hoja encontrado en el pasto Mombasa luego de la remoción de la pastura senescente (Figura 1), hizo que la relación hoja:tallo tuviese valores superiores a los encontrados en el pasto estrella. En pasturas cuyos brotes de crecimiento son más viejos, los porcentajes de hojas son menores, por lo cual la relación hoja:tallo se ve reducida conforme el pasto madura (Simioni et al., 2014).

La remoción de la pastura senescente parece tener un efecto más a largo plazo sobre el pasto Mombasa, lo cual pudo deberse a la arquitectura de la planta (crecimiento en macolla) en comparación con la estrella africana (crecimiento estolonífero). El segado ha mostrado un efecto a más largo plazo con respecto al pastoreo como medio de remoción de senescencia (Tälle et al., 2016). Aunque en este estudio la presión de pastoreo en ambas fincas fue diferente, se considera que no influyó sobre la duración del efecto, pues el pastoreo fue más intensivo en la finca de estrella (45 $\mathrm{m}^{2}$ animal $\left.{ }^{-1}\right)$ con respecto a la finca de Mombasa $\left(125 \mathrm{~m}^{2}\right.$ animal $\left.{ }^{-1}\right)$. El manejo de pasturas debe obtener la mayor producción de forraje con alto valor nutricional, generando una paradoja sobre llenar los requerimientos nutricionales de los animales y las exigencias fisiológicas de las plantas para que la producción no se vea afectada (Simioni et al., 2014).

La práctica de remoción de la pastura senescente disminuyó la producción de biomasa en ambos pastos, siendo de mayor magnitud su efecto en el pasto Mombasa (Cuadro 2 y Figura 3). El pasto Tanzania ha mostrado producciones de biomasa entre 4950 a $6790 \mathrm{~kg} \mathrm{MS} \mathrm{ha}^{-1}$ y entre 4250 a $5370 \mathrm{~kg} \mathrm{MS} \mathrm{ha}^{-1}$ con sistemas de rotación de días fijo (30-35 días) y variable (70 cm altura), respectivamente (Euclides et al., 2014); dichos valores son menores al control y similares a los del tratamiento de este estudio, lo cual coincide con lo encontrado en los componentes estructurales, principalmente material senescente.

El rendimiento anual de biomasa en pasto Mombasa puede ubicarse entre 12100 a $240300 \mathrm{~kg} \mathrm{MS} \mathrm{ha}^{-1}$ al cosecharse de tres a siete semanas (Ramírez-Reynoso et al., 2009). Dichos valores son menores a los obtenidos en este estudio, lo cual puede deberse a la estacionalidad marcada y precipitación menor $\left(<1000 \mathrm{~mm}\right.$ año $\left.{ }^{-1}\right)$ reportadas por Ramírez-Reynoso et al. (2009) con respecto a San Carlos y Turrialba (Quesada, 2007). Dicho comportamiento 
ha sido evaluado en el pasto Bermuda [Cynodon dactylon (L.) Pers. cv. Vaughn's \#1], y ha mostrado variaciones amplias en producción de biomasa (1873-6645 $\mathrm{kg} \mathrm{MS} \mathrm{ha}^{-1}$ ) por efecto del cambio en las condiciones del clima (Gelley et al., 2016).

Debido a que en este estudio no se evaluó el nivel de aprovechamiento de los animales ni se estimó la tasa de crecimiento del pasto a partir de cortes sucesivos bajo una misma altura de remanente, la biomasa anual estimada (Cuadro 2), se puede interpretar como el potencial de acumulación de biomasa. Pasturas con mayor senescencia se asocian a menores consumos, por ello, ajustar la intensidad de pastoreo es uno de los factores que afecta directamente la tasa de acúmulo de forraje (Simioni et al., 2014), resultando en una menor cantidad de material senescente para los siguientes ciclos de rotación (Montagner et al., 2012).

Los pastos perennes con edades de cosecha menores muestran una mayor estabilidad en su tasa de crecimiento con respecto a pastos más maduros (Ramírez-Reynoso et al., 2009). Estos últimos tienen meristemos apicales principalmente en el dosel, por lo que, al remover material a mayor altura se elimina gran parte de ellos, con lo cual se disminuye el vigor de rebrote en el pastoreo siguiente. La gran cantidad de material senescente en las parcelas control limita el ingreso de luz, por lo que hay menor inducción de los brotes basales y menor capacidad de rebrote de la planta (Montagner et al., 2012).

El pasto estrella africana produjo cantidades similares de hoja y tallo (Figura 4) con el tratamiento de remoción, así el nivel de producción de hojas fue levemente superior al indicado por Paris et al. (2016) como óptimo para potencializar la producción de biomasa total $\left(1600 \mathrm{~kg} \mathrm{MS} \mathrm{ha}^{-1}\right)$. Dichos investigadores indican que altos niveles de producción de hojas generan acumulación de material senescente, lo cual explicaría el efecto a corto plazo encontrado en el pasto estrella africana en este estudio. En el caso del pasto Mombasa, el ciclo de recuperación (16 días) menor pudo favorecer que el tratamiento se mantuviese a más largo plazo. Durante el período evaluado, los dos pastos no mostraron floración, por lo que en la planta no experimentaron cambios morfológicos por efecto de la movilización de nutrientes de las hojas a semillas, lo cual genera aumento en la senescencia (Thomas, 2013) y mayor acumulación de tallo (Montagner et al., 2012).

El contenido de MS en el pasto Mombasa tuvo valores menores a los reportados por Patiño-Pardo et al. (2018) para el mismo pasto (19,5-23,7\%) con edades entre 25 y 35 días de recuperación. Debido a que los contenidos de materia seca en los pastos estrella africana $(17,92-22,82 \%)$ y Mombasa $(16,86-23,31 \%)$ variaron posterior a la remoción de material senescente, se considera que el rango encontrado se debe principalmente a los cambios en precipitación durante el período evaluado (febrero a setiembre) (Quesada, 2007).

Los contenidos de proteína del pasto estrella africana en las parcelas control $(8,0-16,4 \%)$ y en el tratamiento de remoción de pasto senescente $(11,7-24,8 \%)$ fueron similares y superiores, respectivamente, a los reportados por Paris et al. (2016) en pasto estrella africana (8,1-14,3\%). Las parcelas control tuvieron contenidos de proteína similares a los encontrados por Gelley et al. (2017) (8,2-14,7 \%) y Gelley et al. (2016) (7,7-15,1\%) en pasto Bermuda con 3 y 4 semanas de rebrote, respectivamente.

El pasto Mombasa en las parcelas control tuvo contenidos inferiores $(6,16 \%)$ a los requeridos por el ganado lechero para mantener un adecuado funcionamiento del rumen (NRC, 2001), lo cual a su vez es un efecto de dilución por la presencia de hojas más viejas, tallos y material senescente (Paris et al., 2016). Los contenidos de proteína del pasto Mombasa en las parcelas control y con remoción de senescencia fueron inferiores (6,28-9,98 \%) a los reportados por Euclides et al. (2014) para el pasto Tanzania (11,3-15,6 \%). Las parcelas control de pasto Mombasa tuvieron contenidos de proteína similares a los reportados por Patiño-Pardo et al. (2018) al aumentar la edad de cosecha de 25 a 45 días con 12,3 y $6,5 \%$, respectivamente.

La remoción de material senescente generó un aumento en la proporción de hojas en ambos pastos (Figura 1), lo que pudo provocar un efecto de concentración de la proteína en menos biomasa. En el proceso de senescencia una hoja pierde cerca del $50 \%$ de su peso, para luego morir e incorporarse al suelo (Simioni et al., 2014). El pasto Mombasa ha mostrado disminuciones cercanas a un $50 \%$ en el contenido proteico al aumentar las edades de 
cosecha de 25 a 45 días (Patiño-Pardo et al., 2018). El aumento en la edad de cosecha en pastos perennes provoca un aumento en la cantidad de material muerto (Ramírez-Reynoso et al., 2009), afectando negativamente la oferta y valor nutricional de materia seca.

El pasto estrella africana en las parcelas control tuvo contenidos de pared celular mayores a estudios previos donde se evaluó el mismo pasto (53,7-65,9\%) con edades de cosecha mayores a cuatro semanas (Paris et al., 2016). Los contenidos de pared celular en el pasto estrella africana fueron similares a los de pasto bermuda con cuatro $(57,6-68,6 \%)$ y tres semanas $(56,2-64,0 \%)$ de rebrote, respectivamente (Gelley et al., 2016; 2017).

La disminución en el contenido de pared celular en ambos pastos puede explicarse por el incremento en la cantidad de hojas (Figuras 1,2), órgano que típicamente ha mostrado contenidos menores de pared celular (Paris et al., 2016). Los contenidos de pared celular en el pasto Mombasa sin remoción de material senescente fueron similares a los reportados por Euclides et al. (2014) para el cv. Tanzania manejado bajo un sistema de rotación de días variable $(71,8-74,5 \%)$ y días fijos $(75,1-75,9 \%)$.

El material senescente en las parcelas control hizo que la oferta de biomasa tuviese un valor nutricional equivalente al de un pasto maduro, tal como reportan Patiño-Pardo et al. (2018) en los contenidos de pared celular para pastos Mombasa (72,6-78,0 \%) y Tanzania (70,1-72,3\%) con 25 y 45 días, respectivamente, lo que a su vez indican que disminuye el consumo de materia seca y el desempeño productivo de los animales.

La digestibilidad de la materia seca y de la pared celular son análisis de gran utilidad para evaluar el valor nutricional de los forrajes, por tratarse de indicadores integrales del aprovechamiento potencial de nutrientes a través del tracto del animal.

La interacción entre el tratamiento y la fecha de muestreo indicó que el efecto de la remoción de senescencia disminuyó con el tiempo. La amplitud en el rango de valores de digestibilidad de la fibra para el tratamiento de remoción se atribuyó a este mismo efecto, ya que ambos pastos tuvieron valores más bajos de digestibilidad de la pared celular en los últimos muestreos. Gelley et al. (2016) obtuvieron valores de digestibilidad de la fibra en pasto Bermuda con cuatro semanas de rebrote menores $(35,3-61,4 \%)$ a los de este estudio, mientras que Gelley et al. (2017) encontraron en pasto Bermuda de tres semanas, digestibilidades de la fibra similares (46,5-54,9\%) a los rangos encontrados en este estudio para las parcelas control $(46,0-67,4 \%)$ y del tratamiento $(42,7-72,5 \%)$. Getachew et al. (2018) indicaron que la digestibilidad de la fibra es un análisis que tiende a variar ampliamente entre materiales vegetales, incluso cuando presentan contenidos de lignina similares.

La remoción de pasto senescente incrementó en promedio ocho y doce puntos porcentuales la digestibilidad de la materia seca de los pastos estrella africana y Mombasa, respectivamente, con respecto al forraje de las parcelas control. Aunque solo en el pasto Mombasa se encontró una interacción significativa entre el tratamiento y la fecha de muestreo, ambos pastos mostraron una tendencia a disminuir con el tiempo, mostrando una caída de catorce y ocho puntos porcentuales en la digestibilidad de la materia seca en los pastos estrella africana y Mombasa, respectivamente.

La digestibilidad de la materia seca encontrada en el pasto Mombasa con remoción de material senescente fue mayor (72,8-79,4 \%) a la encontrada por Euclides et al. (2014) para el pasto cv. Tanzania manejado bajo un criterio de altura de ingreso $(63,2-68,2 \%)$; sin embargo, en las parcelas control $(60,3-65,6 \%)$ fue similar a la reportada por dichos investigadores para Tanzania bajo un sistema de días fijos (61,1-64,6\%). Lo anterior permite evidenciar que el uso de días fijos de rotación en las pasturas hace más probable que se acumule material senescente, debido a las diferencias en crecimiento en el pasto a lo largo del año (Ramírez-Reynoso et al., 2009). Además, es evidencia de la necesidad de desarrollar indicadores confiables para saber el momento óptimo de cosecha, pues incluso la altura del pasto ha mostrado serias deficiencias como predictor del valor nutricional en pastos $\mathrm{C}_{4}$ (Gelley et al., 2017), por lo que otro tipo de herramientas como la fenología deben validarse en pasturas tropicales. Lo anterior refleja la necesidad de hacer ajustes en el sistema de pastoreo, para lograr que los pastos se mantengan en un estado vegetativo con poca acumulación de senescencia, manteniendo área foliar fotosintéticamente activa y permitiendo 
que los animales consuman grandes cantidades de forraje de alto valor nutricional antes de entrar en senescencia (Simioni et al., 2014).

La producción de gas de los pastos estrella africana (14,6-101,7 ml g-1 MS, Figura 9) y Mombasa (23,8-113,1 $\mathrm{ml} \mathrm{g}^{-1} \mathrm{MS}$ ) en este estudio caen dentro del rango (64,3-84,3 $\left.\mathrm{ml} \mathrm{g}^{-1} \mathrm{MS}\right)$ y el promedio (84,1 $\mathrm{ml} \mathrm{g}^{-1} \mathrm{MS}$ ) reportados por Arce-Ramírez (2016) en los mismos pastos, respectivamente. Este autor realizó la incubación de muestras a 24 $\mathrm{h}$, mientras que en este estudio se utilizó un período de $48 \mathrm{~h}$. Investigaciones previas han encontrado diferencias en la producción de gas de acuerdo al tiempo de incubación (Cho et al., 2012; Getachew et al., 2018). Esta metodología se basa en una incubación similar a la realizada para el análisis de digestibilidad de la materia seca, sin embargo, en este caso se mide la producción de gas de forma acumulada estimada en forma de presión y es transformada a ml. En términos generales, otros investigadores han visto que materiales más digestibles tienden a producir un mayor volumen de gas, lo cual tiene que ver tanto con el contenido nutricional del mismo, como con efectos asociativos relacionados con el tipo y cantidad de suplementos brindados al ganado en pastoreo (Xu et al., 2010; Cho et al., 2012).

En este estudio el valor nutricional de ambos pastos influyó sobre la producción de gas, principalmente en el pasto Mombasa, cuyos valores fueron superiores en el tratamiento de remoción de material senescente (Figura 9). El pasto estrella africana tuvo valores similares en las parcelas control y las de remoción, lo cual se atribuyó al efecto temporal del tratamiento que se ha venido mencionando en las variables nutricionales.

Se encontraron correlaciones negativas entre la producción de gas y los componentes de la pared celular (FDN, FDA y lignina) utilizando un período de incubación de 24 h (Arce-Ramírez, 2016). También hubo correlaciones negativas entre el contenido de fibra en detergente neutro y producción de gas in vitro a 12, 24, 36 y $48 \mathrm{~h}$, siendo dichos coeficientes menores conforme se incrementaba el tiempo de incubación (-0,58 a -046) (Coblentz et al., 2013). Ambas investigaciones coinciden en que la fibra detergente neutro es el componente con mayor contribución sobre la producción de gas, siendo inversamente proporcional su efecto. Por otro lado, se ha encontrado una correlación positiva entre la producción de gas y el contenido de carbohidratos no fibrosos (CNF) (Cho et al., 2012).

Ambos pastos mostraron diferencias significativas en el contenido de pared celular en los tratamientos de remoción con respecto a los controles, sin embargo, fue en el pasto Mombasa donde se encontró diferencias en producción de gas in vitro sostenidas en el tiempo. La producción acumulada de gas in vitro en avenas forrajeras con niveles bajos de fibra fueron $14 \mathrm{ml} \mathrm{g}^{-1} \mathrm{MS}$ superiores a la media de avenas forrajeras con alto contenido de fibra (Coblentz et al., 2013). La alfalfa genéticamente modificada para una menor deposición de lignina, ha mostrado producción de gas in vitro levemente superior (5\%) con respecto a cultivares comerciales utilizados como control (Getachew et al., 2018).

Los rangos amplios encontrados en la producción de gas in vitro en ambos pastos se atribuyeron al efecto de la fecha de muestreo. El pasto estrella africana tuvo valores significativamente menores en el primer muestreo (20,9 $\mathrm{ml} \mathrm{g}^{-1} \mathrm{MS}$ ) con respecto a los demás muestreos (77,9 $\left.\mathrm{ml} \mathrm{g}^{-1} \mathrm{MS}\right)$, mientras que en Mombasa los primeros dos muestreos (53,7 ml.g MS ${ }^{-1}$ ) tuvieron valores significativamente menores a los dos últimos $\left(77,9 \mathrm{ml} \mathrm{g}^{-1} \mathrm{MS}\right)$. Se encontraron igualmente rangos amplios con dicha metodología aplicada a avena forrajera (106-195 $\mathrm{ml} \mathrm{g}^{-1} \mathrm{MS}$ ) (Coblentz et al., 2013).

\section{Conclusiones}

La remoción de la pastura senescente permite modificar el crecimiento en pastos perennes tropicales, lo que produce una mayor proporción de hojas y menor de material senescente. Dicho efecto fue más sostenido en el tiempo en el pasto Mombasa con respecto al estrella, lo cual se atribuye a las diferencias en la intensidad de pastoreo en cada finca.

La producción de biomasa en ambos pastos fue menor con la remoción de la pastura senescente, sin embargo, los niveles de producción de materia seca por hectárea se consideran adecuados para pasturas perennes tropicales 
manejadas de forma intensiva. Debido a lo anterior, la técnica de remoción no debería afectar la capacidad de carga en ambos pastos.

La remoción de la pastura senescente produjo un material con menor contenido de pared celular y mayor contenido de proteína, lo cual a su vez produjo forrajes con digestibilidades de la materia seca y de la fibra mayores que en los controles respectivos. Aunque la producción de gas in vitro fue mayor al remover el pasto senescente, debido al valor nutricional superior, un forraje más digestible permite llenar los requerimientos de los animales con menos kg de materia seca de forraje y menor uso de suplementación.

Este estudio demostró que la subutilización de las pasturas tropicales y la acumulación excesiva de material senescente deben considerarse síntomas de degradación de pasturas, por las pérdidas en productividad que generan en los sistemas de ganaderos tropicales. La eficiencia de los sistemas productivos se puede ver optimizada a través de un adecuado manejo de los forrajes, permitiendo una oferta de materia seca de mayor valor nutricional.

\section{Agradecimientos}

Los autores agradecen al productor Daniel Alfaro y al personal del módulo Lechero de la UCR en Turrialba por la colaboración brindada para la realización del presente estudio.

\section{Literatura citada}

ANKOM. 2012. Gas production system operator's manual. ANKOM, Macedon, NY, USA.

AOAC (Association of Official Analytical Chemists). 1990. Official methods of analysis. 15th ed. AOAC Int., Arlington, VA, USA.

Arce-Ramírez, W. 2016. Análisis de correlación y regresión entre la metodología de producción de gas y la ecuación mecanicista del Consejo Nacional de Investigación, EE. UU., (NRC 2001) para determinar el contenido energético in vitro de forrajes. Tesis Lic., Universidad de Costa Rica, San José, CRI.

Coblentz, W.K., S.E. Nellis, P.C. Hoffman, M.B. Hall, P.J. Weimer, N.M. Esser, and M.G. Bertram. 2013. Unique interrelationships between fiber composition, water-soluble carbohydrates, and in vitro gas production for fall-grown oat forages. J. Dairy Sci. 96:7195-7209. doi:10.3168/jds.2013-6889

Cho, A.S., K. Ueda, and S. Kondo. 2012. Evaluation of associative effects on ruminal digestion kinetics between pasture and grains using in vitro gas production method. Anim. Sci. J. 83:650-655. doi:10.1111/j.1740-0929.2011.01003.x

Donaghy, D., and B. Fulkerson. 2001. Principles for developing an effective grazing management system for ryegrass-based pastures. Tasmanian Institute of Agricultural Research, Burnie, Tasmania, AUT.

Dusseux, P., F. Vertès, T. Corpetti, S. Corgne, and L. Hubert-Moy. 2014. Agricultural practices in grasslands detected by spatial remote sensing. Environ. Monit. Assess. 186:8249-8265. doi:10.1007/s10661-014-4001-5

Euclides, V.P.B., D.B. Montagner, G.S. Difante, R.A. Barbosa, and W.S. Fernandes. 2014. Sward structure and livestock performance in guinea grass cv: Tanzania pastures managed by rotational stocking strategies. Sci. Agric. 71:451-457. doi:10.1590/0103-9016-2013-0272

Gelley, C., R.L.G. Nave, and G. Bates. 2016. Forage nutritive value and herbage mass relationship of four warm-season grasses. Agron. J. 108:1603-1613. doi:10.2134/agronj2016.01.0018 
Gelley, C.H., R.L.G. Nave, and G.E. Bates. 2017. Influence of height-based management on forage nutritive value of four warmseason forage grasses. Crop Forage Turfgrass Manag. 3. doi:10.2134/cftm2016.10.0067

Getachew, G., E.A. Laca, D.H. Putnam, D. Witte, M. Mccaslin, K.P. Ortega, and E.J. DePeters. 2018. The impact of lignin down regulation on alfalfa yield, chemical composition, and in vitro gas production. J. Sci. Food Agr. 98:4205-4215. doi:10.1002/jsfa.8942

Holdridge, L.R. 1947. Determination of world plant formations from simple climatic data. Science 105:367-368. doi:10.1126/ science.105.2727.367

Martínez-Méndez, D., J.F. Enríquez-Quiroz, E. Ortega-Jiménez, V.A. Esqueda-Esquivel, A. Hernández-Garay, y J.A.S. Escalante-Estrada. 2016. Rehabilitación de una pradera de pasto Insurgente con diferentes métodos de manejo. Rev. Mex. Cienc. Agríc. 7:1787-1800. doi:10.29312/remexca.v7i8.92

Montagner, D., D. Júnior, B.M. Sousa, H. Vilela, M.C. da-Silveira, V. Euclides, S. da-Silva, and M. Carloto. 2012. Morphogenesis in guinea grass pastures under rotational grazing strategies. R. Bras. Zootec. 41:883-888. doi:10.1590/ S1516-35982012000400008

Murillo, M., E. Herrera, O. Reyes, J. Gurrola, and E. Gutiérrez. 2011. Use in vitro gas production technique for assessment of nutritional quality of diets by range steers. Afric. J. Agric. Res. 6:2522-2526.

NRC (National Research Council). 2001. Nutrient requirements of dairy cattle. 7th rev. ed. The National Academies Press, WA, USA.

Padilla, C., G. Crespo, y Y. Sardiñas. 2009. Degradación y recuperación de pastizales. Rev. Cub. Cienc. Agric. 43:351-354.

Paris, W., R. Tonion, C. Martinello, L.R. Sartor, F.L. de-Paula, and J.G. de-Oliveira. 2016. Productivity and nutritional value of African Star managed with different leaf blade mass. Acta Sci., Anim. Sci. 38:31-36. doi:10.4025/actascianimsci.v38i1.28549

Patiño-Pardo, R.M., R. Gómez Salcedo, y O.A. Navarro Mejía. 2018. Calidad nutricional de Mombasa y Tanzania (Megathyrsus maximus, Jacq.) manejados a diferentes frecuencias y alturas de corte en Sucre, Colombia. Med. Vet. Zootec. 13(1):17-30. doi:10.21615/cesmvz.13.1.2

Quesada, R. 2007. Los bosques de Costa Rica. Presentado en: IX Congreso Nacional de Ciencias. 24 y 25 de agosto, Intituto Tecnológico de Costa Rica. Cartago, CRI.

Ramírez-Reynoso, O., A. Hernández-Garay, S. Carneiro-da-Silva, J. Pérez-Pérez, J.F. Enríquez-Quiroz, A.R. Quero-Carrillo, J.G. Herrera-Haro, y A. Cervantes-Núñez. 2009. Acumulación de forraje, crecimiento y características estructurales del pasto Mombaza (Panicum maximum Jacq.) cosechado a diferentes intervalos de corte. Téc. Pecu. Méx. 47:203-213.

Sade, N., M. Rubio-Wilhelmi, K. Umnajkitikorn, and E. Blumwald. 2017. Stress-induced senescence and plant tolerance to abiotic stress. J. Exp. Bot. 69:845-853. doi:10.1093/jxb/erx235

Sarath, G., L.M. Baird, and R.B. Mitchell. 2014. Senescence, dormancy and tillering in perennial C4 grasses. Plant Sci. 217218:140-151. doi:10.1016/j.plantsci.2013.12.012

Simioni, T.A., A. Hoffmann, F.J. Gomes, C.J. Mousquer, U.H.G. Teixeira, G.A. Fernandes, L.A. Botini, e D.C. de-Paula. 2014. Senescência, remoção, translocação de nutrientes e valor nutritivo em gramíneas tropicais. Pubvet 8:1551-1697. doi:10.22256/pubvet.v8n13.1743

Tälle, M., B. Deák, P. Poschlod, O. Valkó, L. Westerberg, and P. Milberg. 2016. Grazing vs. mowing: A meta-analysis of biodiversity benefits for grassland management. Agric. Ecosyst. Environ. 222:200-212. doi: 10.1016/j.agee.2016.02.008

Thomas, H. 2013. Senescence, ageing and death of the whole plant. New Phytol. 197:696-711. doi:10.1111/nph.12047 
Van-Soest, P.J., and J.B. Robertson. 1985. Analysis of forages and fibrous foods: a laboratory manual for animal science. Cornell University, Ithaca, NY, USA.

Van-Soest, P. J., J. B. Robertson, and B. A. Lewis. 1991. Methods for dietary fiber, neutral detergent fiber, and nonstarch polysaccharides in relation to animal nutrition. J. Dairy Sci. 74:3583-3597. doi:10.3168/jds.S0022-0302(91)78551-2

Villalobos, L., y J. Arce. 2013. Evaluación agronómica y nutricional del pasto Estrella africana (Cynodon nlemfuensis) en la zona de Monteverde, Puntarenas, Costa Rica. I . Disponibilidad de biomasa y fenología. Agron. Costarricense 37:91-101.

Villalobos, L., J. Arce, y R. WingChing. 2013. Producción de biomasa y costos de producción de pastos estrella africana (Cynodon nlemfuensis), kikuyo (Kikuyuocloa clandestina) y ryegrass perenne (Lolium perenne) en lecherías de Costa Rica. Agron. Costarricense 37:91-103.

Villalobos, L., y J. M. Sánchez. 2010. Evaluación agronómica y nutricional del pasto ryegrass perenne tetraploide (Lolium perenne) producido en lecherías de las zonas altas de Costa Rica. I. Producción de biomasa y fenología. Agron. Costarricense 34:31-42.

Villanueva, C., y M. Ibrahim. 2002. Evaluación del impacto de los sistemas silvopastoriles sobre la recuperación de pasturas degradadas y su contribución en el secuestro de carbono en lecherías de altura en Costa Rica. Agrofor. Am. 9(35-36):69-74.

Wilman, D., and V.M.M. Martins. 1977. Senescence and death of herbage during periods of regrowth in ryegrass and red and white clover, and the effect of applied nitrogen. J. Appl. Ecol. 14:615-620. doi:10.2307/2402571

Woo, H.R., C. Masclaux-Daubresse, and P.O. Lim. 2018. Plant senescence: how plants know when and how to die. J. Exp. Bot. 69:715-718. doi:10.1093/jxb/ery011

Xu, M., M. Rinker, K.R. McLeod, and D.L. Harmon. 2010. Yucca schidigera extract decreases in vitro methane production in a variety of forages and diets. Anim. Feed Sci. Technol. 159:18-26. doi:10.1016/j.anifeedsci.2010.05.005 